\title{
The Value of Population Screening in Advancing Personalized Medicine in the Field of Lung Cancer
}

\section{Alice Mogenet \\ Laurent Greillier \\ Pascale Tomasini}

Aix Marseille University, CNRS, INSERM, CRCM, APHM, Multidisciplinary

Oncology \& Therapeutic Innovations

Department, Marseille, France
Correspondence: Pascale Tomasini Service d'Oncologie Multidisciplinaire et Innovations Thérapeutiques, Hôpital

Nord, Chemin des Bourrely, Marseille

Cedex, 13915, France

Tel +33491965901

Fax +33491965902

Email pascale.tomasini@ap-hm.fr

\begin{abstract}
During the past decade, progress has been made in the field of lung cancer molecular biology and onco-immunology, leading to prolonged survival of patients. The combination of increased fundamental knowledge and the pharmaceutical pipeline has allowed the development of various tyrosine kinase inhibitors, targeting numerous molecular alterations. These drugs are now available in daily practice and have transformed survival outcomes for patients harboring EGFR, $A L K$ or ROS1 alterations. Multiple clinical trials are now ongoing in order to increase the number of approved drugs, thus overcoming the issues of rare mutations and tyrosine kinase inhibitors resistance. Immune checkpoint inhibitors development has also changed lung cancer outcomes, but underwhelming response rates highlight the need for immune biomarkers. While PD-L1 expression was the first approved immune biomarker, it has shown several limitations and new biomarkers have to be identified to predict response or resistance to immune checkpoint inhibitors. Testing methods, molecular results and targeted therapeutic schedules will be harmonized in the coming years, with the help of dedicated molecular multidisciplinary boards.
\end{abstract}

Keywords: molecular biology, targeted therapy, lung cancer, immunotherapy, biomarkers

\section{Introduction}

Despite intensive policies trying to reduce tobacco smoking in industrialized countries for the last two decades, lung cancer remains the major cause of cancerrelated death worldwide. ${ }^{1,2}$ It has been a lengthy process trying to endorse the concept of personalized treatment in lung cancer. First, predictive biomarkers of chemotherapy efficacy were studied, such as ERCC1 protein expression, ${ }^{3}$ without real practice-changing consequences. However, the evolution of cancer biology knowledge succeeded in improving lung cancer outcomes, moving from standard chemotherapy regimens to various personalized therapeutic schedules involving targeted therapy, immunotherapy, chemotherapy and antiangiogenics.

Development of the first-generation tyrosine kinase inhibitors (TKIs) erlotinib and gefitinib, is a good illustration of the path trending towards biomarker targeted treatments. These drugs were first developed in non-small cell lung cancer (NSCLC) without patient selection. ${ }^{4,5}$ Then, subgroup analyses highlighted remarkable outcomes among non-smokers, women and Asians, ${ }^{4,6}$ leading to a first step in personalized anticancer treatment. Shortly after, such patients appeared to harbor a relatively high incidence of somatic mutations in the EGFR tyrosine kinase domain. ${ }^{7}$ Further studies easily assessed the superiority of TKIs in second- and first-line settings over chemotherapy in patients harboring EGFR activating 
mutations (Exon 19 or 21) ${ }^{8,9}$ In the light of this paradigm shift and helped by the progressive molecular dismemberment of NSCLC, ${ }^{10}$ several TKIs were developed, targeting major molecular alterations occurring in lung cancer, thus revolutionizing NSCLC outcomes. For instance, a median overall survival of approximately 4 years was achieved for $A L K$-rearranged advanced NSCLC treated with alectinib in the first-line setting. ${ }^{11}$

Meanwhile, a cancer immunology breakthrough led to the development of immune checkpoint inhibitors (ICI), targeting PD-1 or PD-L1, now part of the routine therapeutic toolbox for advanced NSCLC. ${ }^{12-14}$ However, the response rate to ICI is only around $20 \%$ and there was a need to identify a predictive biomarker for immunotherapy. Despite the validation of PD-L1 expression on cancer cells as a routine biomarker in NSCLC, it remains largely disappointing ${ }^{15,16}$ to face further challenges in the immunotherapy field. Tumor immunity cannot be considered in the same way as oncogenic addiction, due to the number of pathways involved, but also due to the concept of progressive mutational burden leading to immune escape and multiway carcinogenesis. ${ }^{17}$

Consequently, molecular screening is now mandatory at the diagnosis of non-squamous NSCLC (and squamous NSCLC in few or never smokers) to personalize NSCLC treatment and enhance disease outcomes. Still, several difficulties will punctuate the coming years, to upgrade the performance of molecular testing, elaborate more and more targeted therapies, for common and especially rare mutations and fill the immunotherapies biomarkers landscape. This article will try to draw actual and further challenges to personalize forever more lung cancer care.

\section{Validated Biomarkers for Lung Cancer: Where are We Now?}

For non-squamous NSCLC, systematic molecular screening at the time of diagnosis is now essential for therapeutic decision-making. Considering the number of targeted therapies available, approved or being developed in clinical trials, repeated molecular screenings are often required to better personalize therapeutic schedules. To date, TKIs are routinely available for five molecular alterations in NSCLC.

The first approved targeted therapies for lung cancer treatment were the first- and second-generation EGFRTKIs. For untreated, advanced, EGFR mutant NSCLC, gefitinib $^{7}$ erlotinib $^{18}$ and afatinib $^{19}$ showed longer progression-free survival (PFS) than the standard platinum-based chemotherapy regimen. These drugs are considered equal in terms of efficacy and toxicity (mostly cutaneous and gastro-intestinal), ${ }^{20,21}$ leaving the choice to clinician discretion. Unfortunately, disease progression always occurs after 9-13 months of treatment. Various mechanisms underlying treatment resistance have progressively been described, the most common being the EGFR exon $20-\mathrm{T} 790 \mathrm{M}$ mutation, in more than $50 \%$ of patients. MET amplifications, KRAS mutations, HER2 amplifications and, although rare, transformations into small cell lung cancer can also be observed as resistance mechanisms. Nevertheless, the resistance pathway remains unknown in about $30 \%$ of cases. ${ }^{22}$ These data have led to the development of osimertinib, a third-generation EGFR-TKI, targeting the T790M mutation. Osimertinib showed superiority as a second-line treatment over chemotherapy in patients harboring T790M mutation. ${ }^{23}$ More recently, the FLAURA trial showed the superiority of osimertinib compared with gefitinib or erlotinib for the first-line treatment of advanced EGFR-mutant NSCLC. The study was positive for progression-free survival (18.9 vs 10.2 months Hazard Ratio (HR) $=0.46[95 \%$ CI $0.37-0.57] \mathrm{p}<0.001),{ }^{24}$ and for overall survival (OS) (38.6 vs 31.6 months $\mathrm{HR}=0.80$ [95\% CI $0.65-1.00] \mathrm{p}=$ $0.046) .{ }^{25}$ In addition, toxicity data were clearly in favor of osimertinib, now approved in the first-line setting.

Soon after the emergence of EGFR-TKIs, targeted therapies were developed for $A L K$-rearranged NSCLC (assessed by immunohistochemistry or in situ hybridization). Crizotinib and ceretinib were the first ALK-TKIs to show superiority over chemotherapy in the first-line setting. ${ }^{26,27}$ However, alectinib showed superiority over crizotinib (PFS 25.7 vs 10.4 months [95\% CI $0.36-0.70], \mathrm{p}<0.001),{ }^{28}$ with outstanding efficacy in patients harboring brain metastases. ${ }^{29}$ Similarly, brigatinib and lorlatinib induced longer PFS than crizotinib (HR respectively 0.43 and 0.28 ). ${ }^{30,31}$ As with $E G F R$-mutant NSCLC patients, $A L K$-rearranged NSCLC patients are ineluctably facing disease progression under targeted therapy, and looking for the resistance mechanism is essential. This resistance mechanism may be $A L K$ dependent ("on-target") or not ("off-target"). If $A L K$ dependent, the resistance mutation must be identified in order to select the second-line TKI. After a first-line treatment with alectinib, the most common resistance mechanism is the G1202R $A L K$-mutation, ${ }^{32}$ warranting a second-line treatment with lorlatinib. Moreover, brigatinib was recently approved after first-line crizotinib, alectinib or ceretinib, 
regardless of the resistance mechanism. ${ }^{33,34}$ Finally, lorlatinib was approved for uncontrolled diseases after a first-line treatment with alectinib or ceritinib or after two lines of ALK-TKIs. ${ }^{35}$ Given its very broad spectrum of activity on various $A L K$ variants, the availability of lorlatinib as a rescue drug is an important advance in the management of $A L K$ rearranged patients.

A ROS1 rearrangement is identified in about $1 \%$ of NSCLC. Crizotinib is approved for both first- and secondline treatment. Indeed, despite the lack of available prospective studies and tiny sizes of the cohorts studied, crizotinib has shown remarkable efficacy in this indication (Objective Response Rate 72\% [95\% CI 58-84]), ${ }^{36}$ even if brain progression remains a struggle in disease control. Recently, entrectinib showed impressive outcomes for ROS1 rearranged patients, especially regarding intracranial and leptomeningal localization (ORR 77.4\% Intracranial ORR $73.9 \%$ ) and is so far recommended in NCNN Guidelines as upfront treatment. ${ }^{37}$ For patients harboring BRAF V600E mutation, the combination of dabrafenib and trametinib was approved but only in a second-line setting. ${ }^{38}$ This combination also showed promising Phase II efficiency data in the first-line setting, showing a response rate of 64\% (95\% CI 46-79) and a PFS of 10.9 months (95\% CI 7.0-16.6). ${ }^{39}$

Along with the previously mentioned biomarkers, representative for classical one-way oncogenic addictions, ICI approval opened new perspectives for personalized medicine. Knowing average response rates to ICI (around $20 \%$ in pretreated patients and up to $50 \%$ in the first-line setting) there is a need for the identification of predictive biomarkers of ICI efficacy. Indeed, tumor cells' PD-L1 expression rate was the first biomarker trying to predict ICI efficacy, conditioning several drug approvals. ${ }^{13,40}$ Moreover, several subgroup analyses based on PD-L1 expression with different cut-offs, reported significant differences in terms of efficacy and survival, ${ }^{12,40,41}$ even if the various measurement skills (platforms and antibodies) must put these data into perspective. ${ }^{42}$ Moreover, the use of PD-L1 expression as a biomarker is still suffering from spatial heterogeneity and remains operator-dependent. Finally, efficacy data among PD-L1 high NSCLC remains highly heterogeneous between several clinical trials and real-life data. ${ }^{15,16}$ Molecular alterations were also studied as ICI biomarkers, with an impressive broad spectrum of impact. $K R A S$ and $B R A F$ mutations, occurring mostly in smokers, appeared to be associated with good response to ICI, especially if combined with TP53 mutation whereas they are associated with ICI resistance if combined with KEAP1 or STK11 mutations. ${ }^{43}$ Moreover, EGFR, HER2, $R E T$ and $A L K$ alterations are associated with poor ICI outcomes, probably because of the small mutational load of these tumors. ${ }^{44}$

\section{Emerging Biomarkers for Lung Cancer: Where are We Going? \\ New Targets}

Rapid progress in the fields of molecular biology and pharmacology enabled the emergence of several molecular-guided treatments in lung cancer. Since most of the frequent molecular alterations can now be targeted by daily care, further challenges will concern sporadic molecular alterations occurring in less than $5 \%$ of NSCLC, with the exception of $K R A S$ mutations, until recently widespread but untargetable. In this subsection, we will discuss approved or studied targeted therapies for those alterations.

cMET amplifications have been described in lung cancer for years now but were difficult to target with multikinase inhibitors such as crizotinib, with fluctuating and disappointing outcomes. ${ }^{45}$ Even if TKIs efficacy is proportional with the amount of $c M E T$ copy number, $c M E T$ really became an oncogenic addiction with the description of MET exon 14 skipping mutations, occurring in about $3 \%$ of NSCLC. Although crizotinib showed an efficacy targeting this alteration, ${ }^{46}$ MET-selective inhibitors significantly improved outcomes in this population. Indeed, capmatinib, tepotinib and savolitinib showed ORR beyond $40 \%$ for pretreated patients, with interesting central nervous system (CNS) efficacy, and median PFS below 10 months. ${ }^{4-49}$

Unlike melanoma, $B R A F$ V600E mutation is relatively rare in NSCLC, occurring in less than $5 \%$ of advanced disease. Even if $B R A F$ single agent inhibition with vemurafenib failed to significantly extend survival despite promising response rates of around $50 \%,{ }^{50}$ the combination of $B R A F$ (dabrafenib) and $M E K$ (trametinib) inhibitors showed significant outcomes improvement in firstand second-line settings with PFS exceeding 12 months. ${ }^{38,39}$ Unfortunately, $B R A F$ non-V600E alterations remain mostly resistant to targeted therapies, and progress will be needed in order to target rare $B R A F$ mutations.

$R E T$ rearrangements involve various fusion partners, $K I F 5 B$ being the most frequent, and are observed in about $1-2 \%$ of NSCLC. Most TKIs studied in this indication were multikinase inhibitors, such as vandetanib or 
cabozantinib, but failed to demonstrate objective response rates better than $40-50 \%$, despite significant toxicities. ${ }^{51,52}$ Recently, a new selective RET inhibitor, selpercatinib, showed remarkable outcomes, especially regarding CNS lesions. The ORR (overall response rate) was $64 \%(95 \%$ CI 54-73) and the objective intracranial response was $91 \%$ (95\% CI 59-100). ${ }^{53}$ Several other TKIs are being developed targeting RET rearrangements, such as BLU-667 (pralsetinib), with promising Phase I trial outcomes. ${ }^{54}$

Molecular rearrangements were also described on the NTRK genes with various fusion partners, resulting in cell cycle dysregulation. Targeting this alteration, the selective pan-TRK inhibitor larotrectinib induced impressive response rates, with ORR $>70 \%$ among several tumor types, leading to the first agnostic pan cancer molecularbased drug approval in the first-line setting. The multikinase inhibitor entrectinib also achieved noteworthy outcomes in this population, corroborating the remarkable oncogenic addiction of NTRK rearrangements. ${ }^{55}$

Although routinely targeted for years now in breast cancer with significant efficacy, anti-HER2 monoclonal antibodies and pan HER TKIs failed to improve outcome of HER2 mutated or amplified NSCLC. ${ }^{56,57}$ Selective drugs were recently diverted from the breast cancer pipeline in order to target HER2 exon 20 mutations in NSCLC. Poziotinib, tarloxotinib and pyrotinib showed promising results in early phase clinical trials. However, the toxicity profile of preliminary data remains significant compared with other TKIs. ${ }^{54}$ Beside TKIs, antibody drug conjugates such as trastuzumab deruxtecan and DS-8201 (telisotuzumab vedotin) showed impressive results in phase I trials including NSCLC patients with ORR over $60 \%{ }^{58,59}$ Giving the molecular similarities between HER2 and $E G F R$ exon 20 insertions, typically resistant to most EGFR-TKIs, the above-mentioned drugs showed comparable results in this indication. Moreover, specific EGFR exon 20 inhibitors such as TAK-788 (mobocertinib), or bispecific antibodies like amivantamab, showed promising results in this indication. ${ }^{60,61}$

Taken together, all the above-mentioned targets do not exceed $20 \%$ of advanced NSCLC. On the other hand, KRAS mutations are the most common alterations observed in NSCLC, in up to $30 \%$ of cases, mostly among current and former smokers. ${ }^{10}$ After years of disillusionment trying to target $K R A S$ with various drugs in NSCLC, promising preliminary results in $K R A S^{\mathrm{G} 12 \mathrm{C}}$ patients revived the hope of considering KRAS as a targetable oncogenic addiction. Sotorasib is a selective and irreversible small $K R A S^{G 12 C}$ inhibitor and showed promising results in phase I study among various tumor types including NSCLC, with a disease control rate of $88.1 \%$ for pretreated patients, and tolerable toxicities. ${ }^{62}$ A phase III study is now ongoing comparing sotorasib efficacy and tolerance to docetaxel. Combination of sotorasib with various other anticancer therapies is also being studied in order to overcome primary and secondary resistance.

\section{Resistance Biomarkers}

Even if the paradigm shift from a common chemotherapy regimen to a permanently more personalized molecular guided treatment significantly improved outcomes of NSCLC, acquired resistance to targeted therapy is inevitable. Molecular biology improvement led to the description of more and more resistance mechanisms, and several drugs are now available to target these acquired alterations. Resistance mechanisms are divided into "on-target", dependent on the baseline altered gene, and "off-target", mostly bypass alterations, independent from the initial alteration. Table 1 summarizes the most commonly described resistance mechanisms and their potential matched treatment, even if not available yet in daily care.

Considering the potential significant efficacy of these beyond progression targeted therapies, iterative molecular screening at the time of progression is now required to identify resistance mechanisms. The identification of an acquired molecular alteration may allow the prescription of an approved targeted treatment, or clinical trial inclusion for new molecules being developed. The exponential number of drugs and combinations in the lung cancer pipeline will inevitably lead to several FDA and EMA registrations in the forthcoming years. Then, the challenge will be to identify the best therapeutic schedule for each alteration in order to personalize sequences to molecular evolution, improving without limits lung cancer outcomes, moving from the current critical prognosis to a chronic controlled disease.

\section{Immune Checkpoint Inhibitors}

Based on the successes of various molecular alterations to predict treatment efficacy, the oncological community initially tried to transpose this approach to identify biomarkers of response for ICI. However, deeply different oncogenic features in underlying response to targeted therapies and to immunotherapy, and the prediction of ICI response became a jigsaw puzzle for scientists. 
Table I Description of the Main Resistance Mechanisms Observed Under TKIs and Corresponding Treatments

\begin{tabular}{|c|c|c|c|c|c|}
\hline Baseline Alteration & Targeted Therapy & Resistance Mechanism & Targeted Therapy & ORR & Reference \\
\hline EGFR activating mutation & $\begin{array}{l}\text { Gefitinib (G) } \\
\text { Erlotinib (E) } \\
\text { Afatinib (A) }\end{array}$ & EGFR Exon 20 T790M & Osimertinib & $71 \%$ & [23] \\
\hline EGFR activating mutation & Osimertinib (O) & EGFR Exon 20 C797S (Cis) & Brigatinib + Cetuximab & $60 \%$ & [63] \\
\hline EGFR activating mutation & Any TKI & MET amplification & $\begin{array}{l}\text { G + Tepotinib } \\
\text { O + Savolitinib } \\
\text { Amivantamab }\end{array}$ & $\begin{array}{c}66,7 \% \\
52 \% \\
\text { NA }\end{array}$ & {$[64,65]$} \\
\hline$A L K$ fusion & $\begin{array}{l}\text { Crizotinib } \\
\text { Alectinib }\end{array}$ & ALK GI202R & $\begin{array}{l}\text { Lorlatinib } \\
\text { Ceritinib }\end{array}$ & $\begin{array}{l}\text { NA } \\
\text { NA }\end{array}$ & \\
\hline$A L K$ fusion & $\begin{array}{l}\text { Crizotinib } \\
\text { Alectinib } \\
\text { Ceritinib }\end{array}$ & ALK LII $196 M$ & Brigatinib & NA & \\
\hline ROSI fusion & Crizotinib & ROSI G2032R & Repotrectinib & $39 \%$ & [66] \\
\hline
\end{tabular}

Abbreviations: ORR, Objective Response rate; TKI, Tyrosine kinase inhibitor; NA, Non available.

Beyond the relative disillusion with PD-L1 expression, several biomarkers may be promising to predict ICI efficacy, as single agent or in combination with chemotherapy or immunotherapy.

Tumor mutational burden (TMB) is described as the total number of mutations per DNA megabase, and is thought to reflect the amount of neoantigens on tumor cells and thus tumor immunogenicity. After the publication of clinical trials with TMB stratification, a pooled analysis showed a linear association between TMB and ORR to anti-PD1 and anti-PDL1, with a correlation coefficient of $0.74(p<0.001) .{ }^{67}$ However, survival data remained disappointing, according for example to the observed benefit from the combination of nivolumab and ipilimumab regardless of TMB cut-off in the CheckMate227 study (HR for death $=0.75$ in the TMB low subgroup and 0.68 in the TMB high subgroup). ${ }^{68}$ Moreover, TMB was developed in the research field using whole exome sequencing techniques, and representative panels and commonly admitted cut-offs are being assessed but still difficult to standardize. Furthermore, quantification of tumor infiltrating lymphocytes (TILs) or other immune cells were studied directly or through different integrative scores with promising results, but failed to establish a robust, reproductible and feasible immune biomarker. Pooled analysis showed that high levels of CD8+, CD3+ and CD4+ TILs are good prognosis biomarkers, with a HR of $0.91,0.77$ and 0.78 for death, respectively. ${ }^{69}$ In addition, growing evidence about interferon gamma pathway implication in PD-L1 expression led to the establishing of transcriptomic signatures, able to predict ICI efficacy in small preliminary cohorts. For example, the 10 -genes IFN- $\gamma$ preliminary signature was significantly associated with best overall response to ICI across various tumor types (melanoma $\mathrm{p}=0.047$; head and neck $\mathrm{p}=0.005) .{ }^{70}$ More anecdotally, methylation profile, previous medications, loss of PTEN expression and microbiota composition are also studied as predictive factors. Besides pathology-based biomarkers, we know that immune tumor burden can induce changes in several circulating parameters, possibly predictive for ICI efficacy. Even if lactate dehydrogenase (LDH), platelet, lymphocytes and neutrophils counts are difficult to interpret because they are also prognostic factors, mathematical modeling seems promising to predict ICI efficacy using routine blood features. The lung immune prognostic index (LIPI), involving dNLR ratio (derived neutrophils/ leukocytes minus neutrophils) and LDH (lactate dehydrogenase) count, appeared to be significantly associated with OS among 466 lung cancer patients treated with ICI (median OS for poor, intermediate and good LIPI were respectively 3, 10 and 34 months $p<0.001) .{ }^{44,71}$ The main research methods for ICI biomarkers are summarized in Figure 1.

Already facing difficulties in prediction of anti-PD1 and anti-PDL1 efficacy, the immune biomarker field will be more and more challenging in the years to come, 


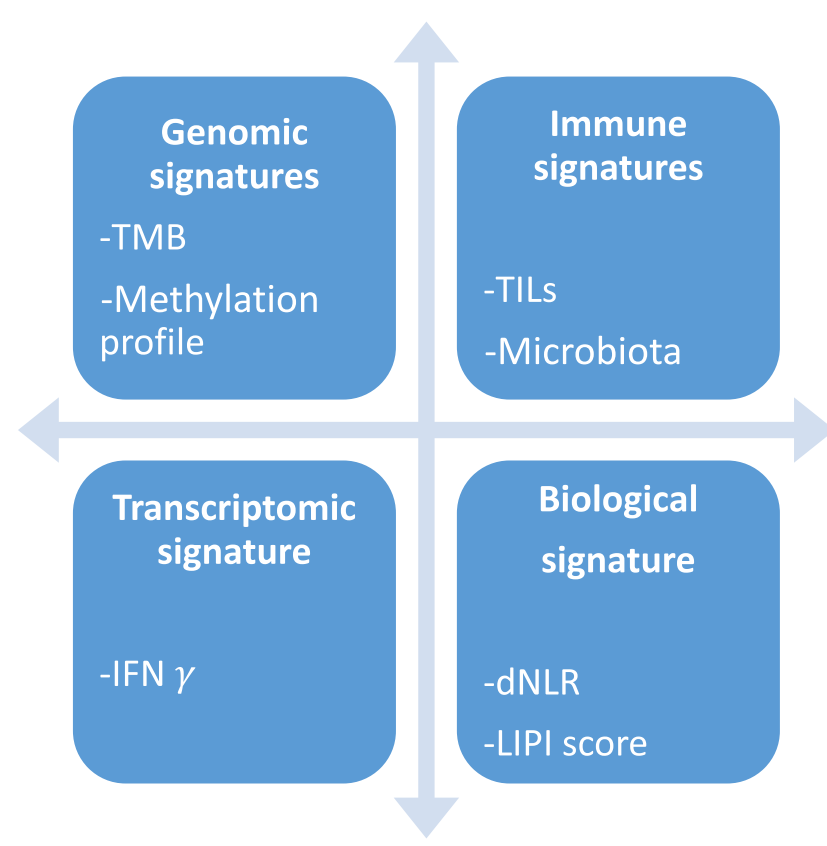

Figure I Main research ways for ICl biomarkers.

Abbreviations: TMB, Tumor Mutational Burden; TILs, Tumor infiltrating lymphocytes; IFN, Interferon; dNLR, Derived neutrophils / leukocytes minus neutrophils; LIPI, Lung immune prognostic index.

considering the number of new molecules and combinations being tested. Beyond lung cancer, the entire medical oncology community is hoping for a personalized immune mapping leading to a personalized multimodal immunotherapy, finally considering immune escape as an oncogenic addiction. As an example, the currently ongoing CARMEN-LC03 phase III trial is assessing efficacy of the antibody drug conjugate SAR408701 targeting CEACAM-5 for CEACAM-5 positive patients (NCT04145956), that can be considered as one of the first targeted immunotherapies.

\section{Face Challenges: How Do We Get There? \\ Testing Strategies}

European guidelines have clearly recommended for years the absolute necessity of molecular screening at diagnosis for all non-squamous carcinomas and never or former smokers (under 15 pack-years) for squamous subtypes. Despite the number of molecular alterations mentioned above, NSCLC ESMO guidelines are only considering $E G F R, A L K, R O S 1$ and $B R A F$ alterations as required at the time of diagnosis. Detection techniques remain each physician's choice depending on local availability of any appropriate, validated method, subject to external quality assurance. ${ }^{72}$ Recently, molecular biology progress allowed many industrialized countries around the world to move from multiple single-gene assays or sequential panels to widespread next-generation sequencing (NGS). The contribution of broader testing in NSCLC was evaluated by clinical trials. First, the MSK-IMPACT trial showed a significant increase of targetable alterations identification with next-generation sequencing of 341 key cancer genes, with a 45 sample gain out of $469^{73,74}$ compared with standard screening. Soon after, the MOSCATO 01 trial assessed the clinical benefit of high-throughput genomic analyses across various tumor types. Of the 843 patients included, a clear benefit from targeted therapy was observed for only 63 patients, nevertheless this was sufficient to significantly improve the PFS ratio, defined as the growth modulation index which compares the PFS under targeted therapy and under the most recent therapy. In the lung cancer cohort, this ratio was considered as positive $(<1.3)$ for only $28 \%$ of patients, thus experiencing a real benefit from the molecular testing. ${ }^{75}$ Finally, results of the umbrella National Lung Matrix Trial (NLMT) were recently published, using genomic data obtained with a 28 genes NGS panel. Despite the number of patients screened and included, the trial failed to show NGS superiority among rare molecular alterations and smokers with Bayesian ORR under $10 \%{ }^{76}$ Considering these data, recommendations were written in 2020 by the ESMO Precision Medicine working group about the use of NGS. In NSCLC, focusing on lung adenocarcinoma, the recommendation for daily practice is to perform tumor multigene NGS to assess level 1 alterations. Larger panels can be used only on the basis of specific agreements with payers taking into account the overall cost of the strategy. NGS can either be done on RNA or DNA, if it includes level 1 fusions in the panel. Recommendations were customized for clinical research centers, labeled as highly recommended to perform multigene sequencing in the context of molecular screening programs in order to increase the access to innovative drugs and to speed up clinical research. $^{77}$

Although large panels are not performed in daily practice, the amount of molecular data available for each patient is growing rapidly, especially in clinical research centers. This information might be hard to interpret for clinicians, and hierarchical classification for the significance and therapeutic implication of molecular alteration is becoming essential. The ESMO ESCAT framework is used to develop the above-mentioned guidelines, but also 
OncoKb

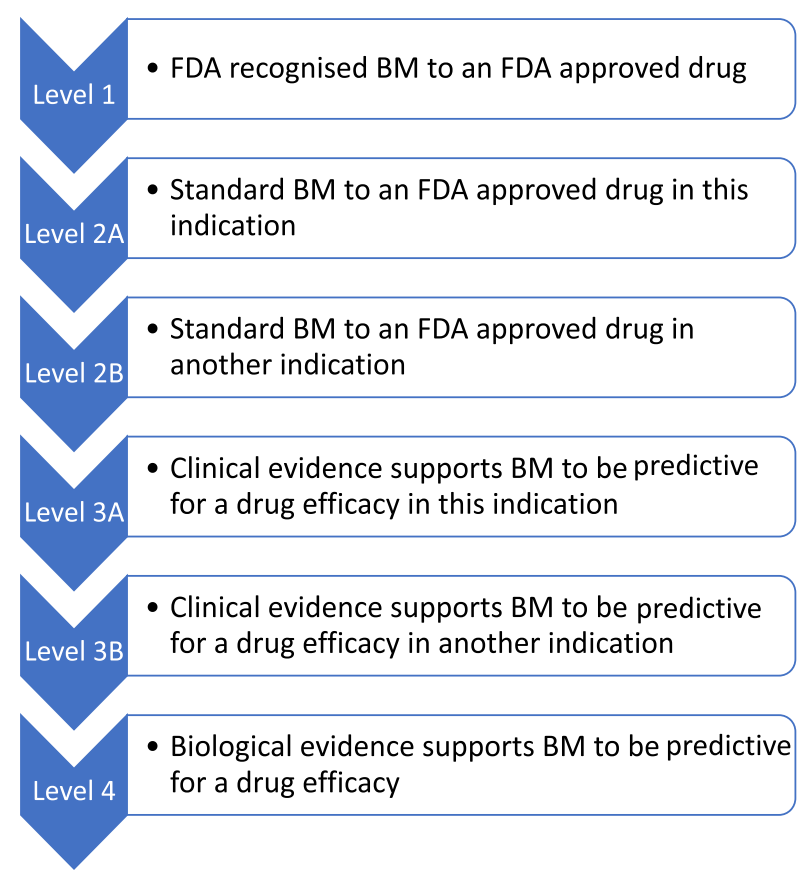

Figure 2 OncoKb and ESCAT classifications of molecular alterations. Abbreviations: BM, Biomarker; FDA, Food and Drug Association.

other knowledge bases such as OncoKb are classifying genomic alterations depending on their potential therapeutic implications. ${ }^{78}$ These two principal scales are reported in Figure 2. In most research centers, multidisciplinary molecular tumor boards (MTB) have been created in order to discuss the clinical implication of molecular data and must be developed. Unfortunately, MTB accessibility is still disappointing, involving less than $50 \%$ of hospitals and $5 \%$ of non-academic hospitals. ${ }^{79}$ Online available knowledge bases can thus be very helpful in order for patients to benefit from molecular screening even in small centers, to identify potentially targetable alterations and address patients in a research center for clinical trial inclusion.

\section{Optimize the Therapeutic Schedule}

This article overviewed the great number of genomic alterations and targeted therapies which are now improving patients' outcomes. To finish, we will discuss the relevance of molecular monitoring in order to personalize treatment sequence. Considering the amount of variants and targeted therapies available for a single gene alteration, and the lack of head to head drug comparison, molecular follow-up is thus a valuable help for clinicians.
ESCAT

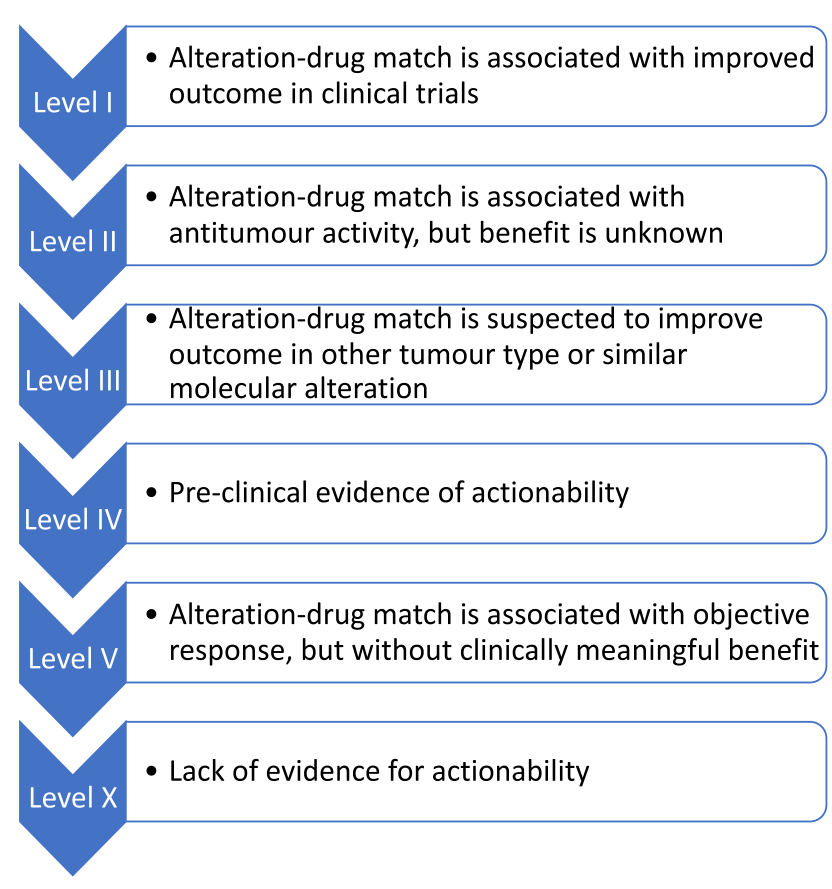

Despite tissue biopsies remaining the gold standard material for molecular analysis, recent technical improvements for liquid biopsies made molecular monitoring easier and useful in daily practice. A recent genome-wide cell-free DNA (cfDNA) fragmentation analysis showed a 91\% sensitivity of detection for circulating tumor DNA (ctDNA) in patients harboring advanced cancer. ${ }^{80}$ On one hand, qualitative detection of molecular alteration allows the identification of resistance mechanisms during targeted therapies. On the other hand, quantitative assessment of cfDNA or of a targeted mutation may enable treatment efficacy and tumor activity, thus helping clinicians to shape the best therapy at every timepoint of the disease, thus avoiding iterative invasive sampling. ${ }^{81}$

If liquid biopsies are obviously useful for the management of targeted therapies, their contribution to predict and follow ICI efficacy remains unclear. After disappointing results concerning the blood evaluation of PD-L1 expression and tumor mutational burden, liquid biopsies may open the way to an indirect immunomonitoring during ICI exposure. Early evaluation of ctDNA response, described as a $>50 \%$ decrease in mutant allele fraction from baseline, was strongly concordant with radiologic response, OS and PFS. ${ }^{82}$ Alternatively, quantification of cfDNA is known to be a surrogate biomarker for STING- 
cGAS pathway activation, which is known to enhance interferon-I production and immune-related cell death, and to be predictive for ICI efficacy. cfDNA quantification could thus be monitored in order to determine the right timing for ICI initiation and infusion intervals. ${ }^{83}$

\section{Conclusion}

Progress in molecular biology and immuno-oncology is leading to the development and approval of a large number of anticancer therapies, especially in the field of lung cancer. Biomarker-based targeted therapies are enhancing both treatment efficacy and tolerance, thus improving lung cancer outcomes. Moreover, the description of various resistance mechanisms and improvement of monitoring techniques is offering a deep personalization of therapeutic schedule for the most commonly described molecular alterations. Nevertheless, the amount of available molecular data has resulted in interpretation issues, and its costeffectiveness has to be assessed. Molecular multidisciplinary boards are thus essential in order to assist clinicians in therapeutic schedule decisions, at diagnosis but also at each step of the disease. Finally, treatment personalization in the field of immunotherapy remains disappointing, with to date the absence of innovative biomarkers applicable in daily practice. If physicians want to go further in personalized medicine, patients' clinical trials inclusion for innovative treatments or biomarkers must be our priority.

\section{Disclosure}

Professor Laurent Greillier reports personal fees, nonfinancial support from Abbvie, Bristol Myers Squibb, Boehringer Ingelheim, Astra Zeneca, Roche, Novartis, MSD, Pfizer, Takeda, outside the submitted work; Dr Pascale Tomasini reports grants, non-financial support from Roche, Astra Zeneca, Takeda, BMS, BI, Novartis, outside the submitted work. The authors report no other conflicts of interest in this work.

\section{References}

1. Barta JA, Powell CA, Wisnivesky JP. Global epidemiology of lung cancer. Ann Glob Health. 2019;85:1.

2. Ferlay J, Colombet M, Soerjomataram I, et al. Estimating the global cancer incidence and mortality in 2018: GLOBOCAN sources and methods. Int J Cancer. 2019;144(8):1941-53. doi:10.1002/ijc.31937

3. Olaussen KA, Mountzios G, Soria J-C. ERCC1 as a risk stratifier in platinum-based chemotherapy for nonsmall-cell lung cancer. Curr Opin Pulm Med. 2007;13(4):284-9.

4. Shepherd FA, Rodrigues Pereira J, Ciuleanu T, et al. Erlotinib in previously treated non-small-cell lung cancer. $N$ Engl $J$ Med. 2005;353(2):123-32.
5. Kim ES, Hirsh V, Mok T, et al. Gefitinib versus docetaxel in previously treated non-small-cell lung cancer (INTEREST): a randomised phase III trial. The Lancet. 2008;372(9652):1809-18.

6. Park K, Goto K. A review of the benefit-risk profile of gefitinib in Asian patients with advanced non-small-cell lung cancer. Curr Med Res Opin. 2006;22(3):561-73.

7. Mok TS, Wu Y-L, Thongprasert S, et al. Gefitinib or carboplatin-paclitaxel in pulmonary adenocarcinoma. $N$ Engl $\mathrm{J}$ Med. 2009;361(10):947-57.

8. Paez JG. EGFR mutations in lung cancer: correlation with clinical response to gefitinib therapy. Science. 2004;304(5676):1497-500.

9. Sequist LV, Martins RG, Spigel D, et al. First-line gefitinib in patients with advanced non-small-cell lung cancer harboring somatic EGFR mutations. J Clin Oncol Off J Am Soc Clin Oncol. 2008;26 (15):2442-9.

10. Barlesi F, Mazieres J, Merlio J-P, et al. Routine molecular profiling of patients with advanced non-small-cell lung cancer: results of a 1-year nationwide programme of the French Cooperative Thoracic Intergroup (IFCT). Lancet Lond Engl. 2016;387(10026):1415-26.

11. Mok T, Camidge DR, Gadgeel SM, et al. Updated overall survival and final progression-free survival data for patients with treatment-naive advanced ALK-positive non-small-cell lung cancer in the ALEX study. Ann Oncol Off J Eur Soc Med Oncol. 2020;31 (8):1056-64.

12. Borghaei H, Paz-Ares L, Horn L, et al. Nivolumab versus docetaxel in advanced nonsquamous non-small-cell lung cancer. $N$ Engl J Med. 2015;373(17):1627-39.

13. Reck M, Rodríguez-Abreu D, Robinson AG, et al. Pembrolizumab versus chemotherapy for PD-L1-positive non-small-cell lung cancer. N Engl J Med. 2016;375(19):1823-33. doi:10.1056/NEJMoa1606774

14. Paz-Ares L, Luft A, Vicente D, et al. Pembrolizumab plus chemotherapy for squamous non-small-cell lung cancer. $N$ Engl $J$ Med. 2018;379(21):2040-51.

15. Aguiar PN, De Mello RA, Hall P, Tadokoro H, de Lima Lopes G. PD-L1 expression as a predictive biomarker in advanced non-smallcell lung cancer: updated survival data. Immunotherapy. 2017;9 (6):499-506.

16. Patel SP, Kurzrock R. PD-L1 expression as a predictive biomarker in cancer immunotherapy. Mol Cancer Ther. 2015;14(4):847-56.

17. Mascaux C, Angelova M, Vasaturo A, et al. Immune evasion before tumour invasion in early lung squamous carcinogenesis. Nature. 2019;571(7766):570-5.

18. Rosell R, Carcereny E, Gervais R, et al. Erlotinib versus standard chemotherapy as first-line treatment for European patients with advanced EGFR mutation-positive non-small-cell lung cancer (EURTAC): a multicentre, open-label, randomised Phase 3 trial. Lancet Oncol. 2012;13(3):239-46.

19. Wu Y-L, Zhou C, Hu C-P, et al. Afatinib versus cisplatin plus gemcitabine for first-line treatment of Asian patients with advanced non-small-cell lung cancer harbouring EGFR mutations (LUX-Lung 6): an open-label, randomised phase 3 trial. Lancet Oncol. 2014;15(2):213-22.

20. Paz-Ares L, Tan E-H, O'Byrne K, et al. Afatinib versus gefitinib in patients with EGFR mutation-positive advanced non-small-cell lung cancer: overall survival data from the phase IIb LUX-Lung 7 trial. Ann Oncol Off J Eur Soc Med Oncol. 2017;28(2):270-7.

21. Urata Y, Katakami N, Morita S, et al. Randomized Phase III study comparing gefitinib with erlotinib in patients with previously treated advanced lung adenocarcinoma: WJOG 5108L. J Clin Oncol Off $J$ Am Soc Clin Oncol. 2016;34(27):3248-57. doi:10.1200/ JCO.2015.63.4154

22. Sequist LV, Waltman BA, Dias-Santagata D, et al. Genotypic and histological evolution of lung cancers acquiring resistance to EGFR inhibitors. Sci Transl Med. 2011;3(75):75ra26. doi:10.1126/ scitranslmed.3002003 
23. Mok TS, Wu Y-L, Ahn M-J, et al. Osimertinib or platinum-pemetrexed in EGFR T790M-positive lung cancer. $N$ Engl J Med. 2017;376(7):629-40. doi:10.1056/NEJMoa1612674

24. Soria J-C, Ohe Y, Vansteenkiste J, et al. Osimertinib in untreated EGFR-mutated advanced non-small-cell lung cancer. $N$ Engl J Med. 2018;378(2):113-25.

25. Ramalingam SS, Vansteenkiste J, Planchard D, et al. Overall survival with osimertinib in untreated, EGFR-mutated advanced NSCLC. $N$ Engl J Med. 2020;382(1):41-50. doi:10.1056/NEJMoa1913662

26. Solomon BJ, Mok T, Kim D-W, et al. First-line crizotinib versus chemotherapy in ALK-positive lung cancer. $N$ Engl J Med. 2014;371 (23):2167-77.

27. Soria J-C, Tan DSW, Chiari R, et al. First-line ceritinib versus platinum-based chemotherapy in advanced ALK-rearranged non-small-cell lung cancer (ASCEND-4): a randomised, open-label, phase 3 study. Lancet Lond Engl. 2017;389(10072):917-29.

28. Peters S, Camidge DR, Shaw AT, et al. Alectinib versus crizotinib in untreated ALK-positive non-small-cell lung cancer. $N$ Engl J Med. 2017;377(9):829-38. doi:10.1056/NEJMoa1704795

29. Gadgeel S, Peters S, Mok T, et al. Alectinib versus crizotinib in treatment-naive anaplastic lymphoma kinase-positive (ALK+) non-small-cell lung cancer: CNS efficacy results from the ALEX study. Ann Oncol Off J Eur Soc Med Oncol. 2018;29(11):2214-22.

30. Camidge DR, Kim HR, Ahn M-J, et al. Brigatinib versus crizotinib in ALK-positive non-small-cell lung cancer. $N$ Engl J Med. 2018;379 (21):2027-39.

31. Shaw AT, Bauer TM, de Marinis F, et al. First-Line lorlatinib or crizotinib in advanced ALK-positive lung cancer. $N$ Engl $\mathrm{J} \mathrm{Med}$. 2020;383(21):2018-29.

32. Gainor JF, Dardaei L, Yoda S, et al. Molecular mechanisms of resistance to first- and second-generation ALK inhibitors in ALK-rearranged lung cancer. Cancer Discov. 2016;6(10):1118-33 doi:10.1158/2159-8290.CD-16-0596

33. Kim D-W, Tiseo M, Ahn M-J, et al. Brigatinib in patients with crizotinib-refractory anaplastic lymphoma kinase-positive non-smallcell lung cancer: a randomized, multicenter Phase II trial. J Clin Oncol Off J Am Soc Clin Oncol. 2017;35(22):2490-8.

34. Descourt R, Perol M, Rousseau-Bussac G, et al. Brigatinib in patients with ALK-positive advanced non-small-cell lung cancer pretreated with sequential ALK inhibitors: a multicentric real-world study (BRIGALK study). Lung Cancer Amst Neth. 2019;136:109-14.

35. Solomon BJ, Besse B, Bauer TM, et al. Lorlatinib in patients with ALK-positive non-small-cell lung cancer: results from a global Phase 2 study. Lancet Oncol. 2018;19(12):1654-67.

36. Shaw AT, Ou S-HI, Bang Y-J, et al. Crizotinib in ROS1-rearranged non-small-cell lung cancer. $N$ Engl J Med. 2014;371(21):1963-71. doi:10.1056/NEJMoa1406766

37. Drilon A, Siena S, Dziadziuszko R, et al. Entrectinib in ROS1 fusion-positive non-small-cell lung cancer: integrated analysis of three Phase 1-2 trials. Lancet Oncol. 2020;21(2):261-70.

38. Planchard D, Besse B, Groen HJM, et al. Dabrafenib plus trametinib in patients with previously treated BRAF(V600E)-mutant metastatic non-small cell lung cancer: an open-label, multicentre phase 2 trial. Lancet Oncol. 2016;17(7):984-93.

39. Planchard D, Smit EF, Groen HJM, et al. Dabrafenib plus trametinib in patients with previously untreated BRAFV600E-mutant metastatic non-small-cell lung cancer: an open-label, phase 2 trial. Lancet Oncol. 2017;18(10):1307-16. doi:10.1016/S1470-2045(17)30679-4

40. Herbst RS, Baas P, Kim D-W, et al. Pembrolizumab versus docetaxel for previously treated, PD-L1-positive, advanced non-small-cell lung cancer (KEYNOTE-010): a randomised controlled trial. Lancet Lond Engl. 2016;387(10027):1540-50.

41. Rittmeyer A, Barlesi F, Waterkamp D, et al. Atezolizumab versus docetaxel in patients with previously treated non-small-cell lung cancer (OAK): a phase 3, open-label, multicentre randomised controlled trial. Lancet Lond Engl. 2017;389(10066):255-65.
42. Kerr KM, Tsao M-S, Nicholson AG, et al. Programmed death-Ligand 1 immunohistochemistry in lung cancer: in what state is this art? $J$ Thorac Oncol Off Publ Int Assoc Study Lung Cancer. 2015;10(7):985-9.

43. Jeanson A, Tomasini P, Souquet-Bressand M, et al. Efficacy of immune checkpoint inhibitors in KRAS-mutant non-small cell lung cancer (NSCLC). J Thorac Oncol Off Publ Int Assoc Study Lung Cancer. 2019;14(6):1095-101.

44. Mezquita L, Auclin E, Ferrara R, et al. Association of the lung immune prognostic index with immune checkpoint inhibitor outcomes in patients with advanced non-small cell lung cancer. JAMA Oncol. 2018;4(3):351-7.

45. Moro-Sibilot D, Cozic N, Pérol M, et al. Crizotinib in c-MET- or ROS1-positive NSCLC: results of the AcSé phase II trial. Ann Oncol Off J Eur Soc Med Oncol. 2019;30(12):1985-91.

46. Drilon A, Clark JW, Weiss J, et al. Antitumor activity of crizotinib in lung cancers harboring a MET exon 14 alteration. Nat Med. 2020;26(1):47-51.

47. Wolf J, Seto T, Han J-Y, et al. Capmatinib in MET Exon 14-mutated or MET-amplified non-small-cell lung cancer. $N$ Engl J Med. 2020;383(10):944-57. doi:10.1056/NEJMoa2002787

48. Paik PK, Felip E, Veillon R, et al. Tepotinib in non-small-cell lung cancer with MET Exon 14 skipping mutations. $N$ Engl J Med. 2020;383(10):931-43. doi:10.1056/NEJMoa2004407

49. Lu S, Fang J, Li X, et al. Phase II study of savolitinib in patients (pts) with pulmonary sarcomatoid carcinoma (PSC) and other types of non-small cell lung cancer (NSCLC) harboring MET exon 14 skipping mutations (METex14+). J Clin Oncol. 2020;38 (15_suppl):9519-9519. doi:10.1200/JCO.2020.38.15_suppl.9519

50. Mazieres J, Cropet C, Montané L, et al. Vemurafenib in non-smallcell lung cancer patients with BRAFV600 and BRAFnonV600 mutations. Ann Oncol Off J Eur Soc Med Oncol. 2020;31(2):289-94.

51. Yoh K, Seto T, Satouchi M, et al. Vandetanib in patients with previously treated RET-rearranged advanced non-small-cell lung cancer (LURET): an open-label, multicentre phase 2 trial. Lancet Respir Med. 2017;5(1):42-50.

52. Drilon A, Rekhtman N, Arcila M, et al. Cabozantinib in patients with advanced RET-rearranged non-small-cell lung cancer: an open-label, single-centre, phase 2, single-arm trial. Lancet Oncol. 2016;17 (12):1653-60.

53. Drilon A, Oxnard GR, Tan DSW, et al. Efficacy of selpercatinib in RET fusion-positive non-small-cell lung cancer. $N$ Engl $\mathrm{J}$ Med. 2020;383(9):813-24.

54. Subbiah V, Gainor JF, Rahal R, et al. Precision targeted therapy with BLU-667 for RET-driven cancers. Cancer Discov. 2018;8 (7):836-49.

55. Haratake N, Seto T. NTRK fusion-positive non-small-cell lung cancer: the diagnosis and targeted therapy. Clin Lung Cancer. 2021;22(1):1-5.

56. Jebbink M, de Langen AJ, Boelens MC, Monkhorst K, Smit EF. The force of HER2 - A druggable target in NSCLC? Cancer Treat Rev. 2020;86:101996.

57. Robichaux JP, Elamin YY, Tan Z, et al. Mechanisms and clinical activity of an EGFR and HER2 exon 20-selective kinase inhibitor in non-small cell lung cancer. Nat Med. 2018;24(5):638-46.

58. Tsurutani J, Iwata H, Krop I, et al. Targeting HER2 with trastuzumab deruxtecan: a dose-expansion, Phase I study in multiple advanced solid tumors. Cancer Discov. 2020;10(5):688-701.

59. Smit EF, Nakagawa K, Nagasaka M, et al. Trastuzumab deruxtecan (T-DXd; DS-8201) in patients with HER2-mutated metastatic non-small cell lung cancer (NSCLC): interim results of DESTINY-Lung01. J Clin Oncol. 2020;38(15_suppl):9504-9504. doi:10.1200/JCO.2020.38.15_supp1.9504

60. Riely GJ, Neal JW, Camidge DR, et al. Activity and safety of mobocertinib (TAK-788) in previously treated non-small cell lung cancer with EGFR Exon 20 insertion mutations from a Phase I/II trial. Cancer Discov. 2021;11(7):1688-1699. doi:10.1158/2159-8290. CD-20-1598 
61. Yun J, Lee S-H, Kim S-Y, et al. Antitumor activity of Amivantamab (JNJ-61186372), an EGFR-MET bispecific antibody, in diverse models of EGFR Exon 20 insertion-driven NSCLC. Cancer Discov. 2020;10(8):1194-209.

62. Hong DS, Fakih MG, Strickler JH, et al. KRASG12C inhibition with sotorasib in advanced solid tumors. $N$ Engl J Med. 2020;383 (13):1207-17. doi:10.1056/NEJMoa1917239

63. Wang Y, Yang N, Zhang Y, et al. Effective treatment of lung adenocarcinoma harboring EGFR-activating mutation, T790M, and cis-C797S triple mutations by brigatinib and cetuximab combination therapy. J Thorac Oncol Off Publ Int Assoc Study Lung Cancer. 2020;15(8):1369-75.

64. Wu Y-L, Cheng Y, Zhou J, et al. Tepotinib plus gefitinib in patients with EGFR-mutant non-small-cell lung cancer with MET overexpression or MET amplification and acquired resistance to previous EGFR inhibitor (INSIGHT study): an open-label, phase 1b/2, multicentre, randomised trial. Lancet Respir Med. 2020;8(11):1132-43.

65. Sequist LV, Han J-Y, Ahn M-J, et al. Osimertinib plus savolitinib in patients with EGFR mutation-positive, MET-amplified, non-smallcell lung cancer after progression on EGFR tyrosine kinase inhibitors: interim results from a multicentre, open-label, phase $1 \mathrm{~b}$ study. Lancet Oncol. 2020;21(3):373-86.

66. Drilon A, Ou S-HI, Cho BC, et al. Repotrectinib (TPX-0005) is a next-generation ROS1/TRK/ALK inhibitor that potently inhibits ROS1/TRK/ALK solvent- front mutations. Cancer Discov. 2018;8 (10):1227-36.

67. Yarchoan M, Hopkins A, Jaffee EM. Tumor mutational burden and response rate to PD-1 inhibition. $N$ Engl J Med. 2017;377 (25):2500-1. doi:10.1056/NEJMc1713444

68. Hellmann MD, Paz-Ares L, Bernabe Caro R, et al. Nivolumab plus ipilimumab in advanced non-small-cell lung cancer. $N$ Engl J Med. 2019;381(21):2020-31. doi:10.1056/NEJMoa1910231

69. Zeng D-Q, Yu Y-F, Ou Q-Y, et al. Prognostic and predictive value of tumor-infiltrating lymphocytes for clinical therapeutic research in patients with non-small cell lung cancer. Oncotarget. 2016;7 (12):13765-81.

70. Ayers M, Lunceford J, Nebozhyn M, et al. IFN- $\gamma$-related mRNA profile predicts clinical response to PD-1 blockade. J Clin Invest. 2017;127(8):2930-40. doi:10.1172/JCI91190

71. Gu X, Sun S, Gao X-S, et al. Prognostic value of platelet to lymphocyte ratio in non-small cell lung cancer: evidence from 3430 patients. Sci Rep. 2016;6:23893.
72. Planchard D, Popat S, Kerr K, et al. Metastatic non-small cell lung cancer: ESMO Clinical Practice Guidelines for diagnosis, treatment and follow-up. Ann Oncol Off J Eur Soc Med Oncol. 2018;29 (Supplement_4):iv192-237.

73. Cheng DT, Mitchell TN, Zehir A, et al. Memorial Sloan Kettering-integrated mutation profiling of actionable cancer targets (MSK-IMPACT): a hybridization capture-based next-generation sequencing clinical assay for solid tumor molecular oncology. J Mol Diagn JMD. 2015;17(3):251-64.

74. Jonna S, Subramaniam DS. Molecular diagnostics and targeted therapies in non-small cell lung cancer (NSCLC): an update. Discov Med. 2019;27(148):167-70.

75. Massard C, Michiels S, Ferté C, et al. High-throughput genomics and clinical outcome in hard-to-treat advanced cancers: results of the MOSCATO 01 trial. Cancer Discov. 2017;7(6):586-95.

76. Middleton G, Fletcher P, Popat S, et al. The national lung matrix trial of personalized therapy in lung cancer. Nature. 2020;583 (7818):807-12.

77. Mosele F, Remon J, Mateo J, et al. Recommendations for the use of next-generation sequencing (NGS) for patients with metastatic cancers: a report from the ESMO Precision Medicine Working Group. Ann Oncol Off J Eur Soc Med Oncol. 2020;31(11):1491-505.

78. Chakravarty D, Gao J, Phillips SM, et al. OncoKB: a precision oncology knowledge base. JCO Precis Oncol. 2017;2017.

79. van der Velden DL, van Herpen CML, van Laarhoven HWM, et al. Molecular Tumor Boards: current practice and future needs. Ann Oncol Off J Eur Soc Med Oncol. 2017;28(12):3070-5.

80. Cristiano S, Leal A, Phallen J, et al. Genome-wide cell-free DNA fragmentation in patients with cancer. Nature. 2019;570(7761):385-9.

81. Sorber L, Zwaenepoel K, Deschoolmeester V, et al. Circulating cell-free nucleic acids and platelets as a liquid biopsy in the provision of personalized therapy for lung cancer patients. Lung Cancer Amst Neth. 2017;107:100-7.

82. Goldberg SB, Narayan A, Kole AJ, et al. Early assessment of lung cancer immunotherapy response via circulating tumor DNA. Clin Cancer Res Off J Am Assoc Cancer Res. 2018;24(8):1872-80.

83. Sicard G, Fina F, Fanciullino R, Barlesi F, Ciccolini J. Like a rolling stone: sting-cgas pathway and cell-free DNA as biomarkers for combinatorial immunotherapy. Pharmaceutics. 2020;12:8.
Pharmacogenomics and Personalized Medicine

\section{Publish your work in this journal}

Pharmacogenomics and Personalized Medicine is an international, peer-reviewed, open access journal characterizing the influence of genotype on pharmacology leading to the development of personalized treatment programs and individualized drug selection for improved safety, efficacy and sustainability. This journal is indexed on the American Chemical Society's Chemical Abstracts Service (CAS). The manuscript management system is completely online and includes a very quick and fair peer-review system, which is all easy to use. Visit http://www.dovepress.com/testimonials.php to read real quotes from published authors. 